\title{
REVIEW
}

\section{An evolutionary paradigm for carcinogenesis?}

\author{
P Vineis, G Matullo, M Manuguerra
}

J Epidemiol Community Health 2003;57:89-95

Mutations seem to be only one of the mechanisms involved in carcinogenesis; selection of mutated clones is a second crucial mechanism. An evolutionary (darwinian) theory of carcinogenesis can be useful to explain some contradictory observations of epidemiology, and to provide a common theoretical framework for carcinogenesis. In both the selection of species and in carcinogenesis (selection of mutated cells), mutation and selection can be interpreted as necessary and insufficient causes. Selection presupposes competition among clones-that is, survival advantage of the mutated species; without selective forces a mutation is mute, while the lack of mutations makes selective advantage impossible. The identification of carcinogen related fingerprints is ambiguous: it can suggest both a genuine mutational hotspot left by the carcinogenic stimulus (like in tobacco related p53 mutations), and selective advantage of clones whose mutations seem to be not exposure specific (like in the case of aflatoxin). We present several examples of exposures that can increase the risk of cancer in humans not via mutations but through a putative mechanism of clone selection.

See end of article for authors' affiliations

Correspondence to: Dr P Vineis, Servizio di Epidemiologia dei Tumori, CPO-Centro di Riferimento per l'Epidemiologia e la Prevenzione Oncologica in Piemonte, Via Santena 7,

10126 Torino, Italy;

vineis@inrete.it

\section{GENES AND CANCER} considered from three different viewpoints: (a) cancer as a consequence of germline mutations or polymorphisms; (b) cancer as a consequence of mutations in somatic cells; (c) cancer as a consequence of germline or somatic mutations followed by clonal selection.

Concerning the first viewpoint, a simplistic interpretation of carcinogenesis has tended to attribute to genetic predisposition (germline mutations or variants) a role that it only rarely has. Single highly penetrant mutations in "cancer genes" explain only a small proportion of cancers (around 5\%). This consideration arises both from empirical observation and from general scientific knowledge. Highly penetrant gene mutationsthat confer an exceptionally high risk of cancer in the carriers-represent the tail of a distribution that includes: (a) common variants in the same cancer genes (polymorphisms) that have a less disruptive effect on the protein function, and (b) mutations in genes that are more indirectly involved in the cancer process. There is increasing evidence in favour of both categories. ${ }^{1}$
The relation between genes and cancer has been
In general, genetic determinism has been criticised on several grounds:

- Humans, mice, and other animals have about the same estimated number of expressed genes

- humans and chimpanzees share $98 \%$ of the genome

- the sequence information in DNA is insufficient to determine how gene products interact to produce a whole organism

- genetic pathways completely specify organismal function only in rare cases - that is, monogenic diseases like sickle cell anaemia, muscular distrophy or Huntington corea, in which the cell has no compensatory mechanism to overcome the metabolic impairment and the enviromental influences are nil; these are the only (rare) instances in which a "one gene-one disease" paradigm is valid. ${ }^{2}$

Therefore, the attention should be more on somatic mutations than on genetic predisposition, at least from a clinical and public health perspective.

\section{MUTATIONS AND CARCINOGENESIS}

The predominant view of carcinogenesis confers a central role to somatic mutations. This interpretation is based on both the history of chemical carcinogenesis and the theory of mutational "hotspots" or fingerprints. ${ }^{3}$ The first evidence that chemicals induce cancer came from both epidemiological observation (scrotal cancer in chimney sweeps described by Percival Pott) and experiments in animals (the famous experiments conducted by painting coal tar on the ears of rabbits by Ishikawa and colleagues). The first chemical carcinogens to be isolated were polycyclic aromatic hydrocarbons in the 1920s, by Kennaway and colleagues at the London Institute for Cancer Research. ${ }^{4}$ Polycyclic aromatic hydrocarbons were considered to be responsible for the carcinogenicity of coal tar. In the 1960 Brookes and Lawley discovered that carcinogens react with DNA. ${ }^{5}$

More recently, a strong confirmation of the fact that cancer is attributable to mutations-induced by chemicals or other agents-has come from the identification of apparent "fingerprints" left by chemical carcinogens in specific codons of genes deeply involved in the cell cycle such as $p 53$. The most famous of fingerprints was that described in codon 249 of $p 53$ (a G to T transition), allegedly left by aflatoxin in hepatocellular carcinoma cells. ${ }^{6}$ The immediate interpretation of this observation, made by two independent groups of researchers in different parts of the world (Turkey and China), where aflatoxin exposure is endemic, was that each carcinogen might leave its characteristic "signature" in DNA, namely in genes that 
play a crucial part in carcinogenesis. The "fingerprint theory" - at least in its strongest form-implies (a) that cancer is essentially attributable to mutations, and (b) that each specific carcinogen leaves a typical signature on DNA. ${ }^{3}$

However, this interpretation has received rather serious disconfirmation in a study by Denissenko and colleagues. ${ }^{7}$ They incubated cells with aflatoxin $\mathrm{Bl}$ and then studied the types of DNA adducts induced in $p 53$ (adducts express the amount of a chemical bound to DNA in a specific site). They observed that adducts were mainly in sites different from codon 249 , the one that the "fingerprint" theory implicated. In addition, the expected adducts in codon 249 were rapidly repaired $(50 \%$ in seven hours). Therefore, the possibility that aflatoxin exerts its carcinogenic activity by leaving a signature in a specific codon and with a specific mechanism in $p 53$ was considerably weakened. Nevertheless, some aspects of the fingerprint hypothesis remain worthwhile. Smokers with lung cancer show a pattern of mutations in $p 53$ that is considerably different from that of non-smokers. The same group of Denissenko has shown that in the very same sites of $p 53$, where typical mutations are found in smokers with lung cancer, the carcinogenic chemical benzo(a)pyrene also forms adducts. ${ }^{8}$

Is the fingerprint theory true in some occasions and not in others? And what is the explanation for the discrepancy between the original observations in hepatocellular carcinoma and the experiments on aflatoxin by Denissenko? The interpretation of the Denissenko group is that, in fact, the mutations we observe in cancer cells are not those originally induced by the carcinogen; rather, they are an expression of a complex process of selection the cells have undergone before the clinically overt cancer arises. More precisely, the interpretation is that additional factors-other than mutational events-have led to the selection of some cellular clones characterised by the apparent fingerprint; one such additional factor in liver carcinogenesis might be infection with the hepatitis B virus.

Concerning the fingerprint left by tobacco smoke, a discussion has arisen recently. In a paper based on a mathematical analysis of the IARC database on $p 53$ mutations, Rodin and Rodin have claimed that there was no association between mutational hotspots and smoking habits. ${ }^{9}$ However, a more accurate re-analysis done by Hainaut and Pfeifer has revealed that Rodin and Rodin's claim was not supported: after selection of the relevant subset of cases, the mutational spectra were different in smokers and non-smokers at a high level of statistical significance. ${ }^{10}$ In fact, G to T transversions of $p 53$ have been linked to a molecular signature of tobacco smoke mutagens in smoking associated lung cancers for the following reasons: (a) PAHs are prominent carcinogens in tobacco smoke and they produce predominantly this type of mutation; (b) PAH adducts are present in DNA extracted from human tissues exposed to tobacco smoke; (c) there is an increased frequency of $\mathrm{G}$ to $\mathrm{T}$ transversions in lung cancers from smokers compared with lung cancers from non-smokers; (d) a non-transcribed strand bias of $G$ to $T$ transversions can be attributed to preferential repair of adducts on the transcribed strand.$^{10}$ Mutations of $p 53$ are considered to be an early event in lung carcinogenesis, as they are already present in preneoplastic lesions of the lung.

\section{THE ROLE OF CLONAL SELECTION}

Mutations seem to be only one of the mechanisms involved in carcinogenesis. In fact, if we consider the large number of cell divisions that occur daily in our body, and the frequency of mutations-attributable, for example, to errors in DNA replication-, cancer should be extremely frequent. "Spontaneous" mutations (the inverted commas are justified as we cannot separate mutations that are really spontaneous from those induced by the environment) are in the order of one per million copies of a gene. As genes are about 30 000, and cells of the bone marrow or the small intestine replicate themselves (and their genes) at a rate of $10^{11}$ per day, we should have a large number of tumours arising every day. A further piece of evidence that mutations are not enough comes from the observation that in our skin epithelium there are 50 clones $/ \mathrm{cm}^{2}$ that carry $p 53$ mutations, each clone consisting of 60-30 000 cells. ${ }^{11}$ Again, why don't we develop cancer more easily?

The explanation seems to lie in the mechanisms that allow us to get rid of damaged DNA, either by repairing it or by directly eliminating the mutated cells by apoptosis (programmed cell death). Interestingly, time and dose are crucial in the relation between a carcinogenic exposure and the onset of cancer. In a series of experiments, Iversen treated the skin of mice with a single dose of $51.2 \mu \mathrm{g}$ of DMBE, then with 25.6 $\mu \mathrm{g}$ in two doses, with $10 \mu \mathrm{g}$ six times, with $2.6 \mu \mathrm{g} 20$ times, and $1 \mu \mathrm{g} 50$ times. $^{12}$ The observed to expected ratio for tumours was 0.04 for acetone (that is, in the absence of the carcinogen), $0.62,1.74,2.93,7.04$, and 7.95, respectively, with increasingly fractionated doses (one is the average ratio in the whole group). These experiments suggest that repeated small doses are more powerful in inducing cancer than the same total amount administered once. A very similar observation has been made in humans exposed to tobacco smoke: the excess incidence of lung cancer in moderate smokers was 0.005 for those who smoked 15 years, 0.1 for those who smoked 30 years, and 0.5 for 45 years. In heavy smokers, the same figures were $0.01,0.2$, and 1 . In both moderate and heavy smokers, the excess incidence rose by 100 times when duration increased by three times-that is, the risk was approximately the fourth power of duration. ${ }^{13}$ These observations suggest that repeated carcinogenic stimuli are likely to overcome defensive processes, such as DNA repair and apoptosis. However, this is probably not the only explanation. In Iversen's experiments, in fact, another strong determinant of the tumour yield was the length of the intervals between doses: the longer the interval, the greater the cancer yield.

There is a similarity between the latter observation and a totally different field of biology, the theory of evolution. Differences might conceal more important similarities. According to the neo-darwinian interpretation, the appearance of a new species is the consequence of two distinct events (or processes), mutation and selection. In the presence of environmental changes, the carriers of certain mutations will have a selective advantage that will lead to the appearance of a new species, especially if the population originating from the mutated carrier remains isolated enough from the ancestor species in terms of time and space. From our perspective, what is interesting is that also in "speciation" a certain time lag between mutation and selection is necessary for selection to be effective, ${ }^{14}$ probably because the appearance of the new species is related to the existence of a sufficiently large population of individuals (a critical mass) to make the species really competitive.

One property of simple organisms, such as bacteria, is that when they are incubated in a stressful environment (for example, lacking the usual nutritional substrates) they quickly develop a large number of gene mutations-that is, they acquire a "mutator phenotype", characterised by extreme genomic instability. ${ }^{15}$ This can be interpreted as an evolutionary feature, perhaps related to the fact that a large number of mutations will increase the probability of finding the one that allows the bacterium to adapt to the changing environment. It is hypothesised that the genomic instability characterising stressful (toxic) environments is related to a loss of the usual DNA repair mechanisms. Similar experiments have been done in mice. Mice that were deficient in $\mathrm{O}^{6}$-methylguanine DNA-methyltransferase (MGMT) and in MMR (mismatchrepair of DNA) were resistant to the toxic effects of MNU (methylnitrosourea, a potent methylating agent), but they developed a large number of tumours. These findings have 
Table 1 Case-control studies on cancer and WBC-DNA adducts (P32-postlabelling or enzyme linked immunosorbent assay, ELISA) (from Vineis and Perera ${ }^{23}$ )

\begin{tabular}{|c|c|c|}
\hline Authors and method & Type of cancer & OR $(95 \% \mathrm{Cl})$ \\
\hline $\begin{array}{l}\text { Peluso et al, } 2000 \\
\text { Quartiles of DNA adducts }\end{array}$ & bladder & 5.25 (2.2 to 12.4$)$ \\
\hline$<0.1$ & & $1.0-$ \\
\hline $0.1-0.23$ & & 3.0 (1.45 to 6.1$)$ \\
\hline $0.24-0.51$ & & $5.4(2.5$ to 11.7$)$ \\
\hline$>0.51$ & & 7.9 (3.4 to 18.4$)$ \\
\hline Vulimiri et al, 2000 & lung & $\begin{array}{l}\text { mean adducts } \\
\text { (per } 10^{8} \text { nucleotides): } \\
\text { cases } 6.03 \text { (1.16 SE) } \\
\text { controls } 2.80 \text { (0.36 SE) (c) }\end{array}$ \\
\hline Hou et al, 1999 & lung & $\begin{array}{l}4.1 \times 10^{-8} \\
\text { in both cases and controls }\end{array}$ \\
\hline Li et al, 1996 & lung & 6.4 (1.3 to 29.4$)$ \\
\hline Tang et al, 1995, ELISA & lung & 7.7 (1.7 to 34$)$ \\
\hline
\end{tabular}

Adducts are expressed as RAL $\times 10^{-8}$; estimates adjusted for age, smoking, gender, ethnicity, and (Peluso) fruit and vegetable intake. OR, odds ratio; $\mathrm{Cl}$, confidence intervals.

been interpreted as suggesting that cells undergoing methylation by a methylating agent (that is, a strong mutagen) show a paradoxical response, because the mutagenic environment also potentiates the growth advantage of DNA repair deficient cells. ${ }^{16}$ It should be emphasised that the experiments described were carried out at high, toxic doses, not relevant to usual human exposures.

Other experiments in Escherichia coli cultures $^{17}$ have suggested recently that a transient increase in mutator frequencies should be a common event in natural asexual populations subjected to sporadic adaptive evolution. Pathogenic bacteria-as compared with non-pathogenic-show a high frequency of mutators, suggesting that the evolution of increased mutation rates is relevant to the evolution of parasites. ${ }^{18}$ Also inducible mechanisms that cause a transient mutator phenotype have been described in response to stress. ${ }^{19}$

\section{APC MUTATIONS AND COLORECTAL CANCER}

Mutations that provide a selective advantage are certainly important for tumorigenesis; this implies that a powerful evolutionary force is expected to be natural selection, which acts to increase the frequency of advantageous alleles in the population. ${ }^{20}$ For instance, in the well recognised stepwise model of colorectal cancer, mutations of APC tend to occur before two mutations in mismatch repair genes (MMR) have occurred. The spectrum of APC mutations in RER+ (replication error prone) tumours does not reflect an underlying preexisting defect in $\mathrm{MMR}^{21}$ showing that the APC mutations occurred when MMR was normal. In patients with HNPCC (hereditary non-polyposis colon cancer), by contrast, one MMR mutation is inherited. These patients have a raised mutation rate and the second hit on MMR tends to take place before the first hit at APC. HNPCC patients have about the same number of colorectal tumours as the general population, whereas FAP patients (with a germline APC mutation) usually develop thousands of colorectal tumours. After two APC mutations, expansion of the colorectal tumour clone occurs. Subsequent rounds of clonal expansion, mutation, and selection repeat this process, leading to the stepwise, highly complex, progression of colorectal tumours.

Excision repair mutations rarely occur in sporadic cancers, although they lead to an increased cancer risk through genomic instability when two mutant alleles are present in the germline cells. This fact alone and the comparison between FAP and HNPCC means that there is no simple association between increased mutation rates (or genomic instability) and tumorigenesis. Rather, it is the interplay among mutations, impairment of DNA repair and cell cycle checkpoints, clonal expansion, and selection processes that eventually yields the neoplastic phenotype.

\section{AN EXAMPLE FROM A NON-MALIGNANT DISEASE: PAROXYSMAL NOCTURNAL HAEMOGLOBINURIA}

Paroxysmal nocturnal haemoglobinuria (PNH) is an acquired stem cell disorder characterised by intravascular haemolysis, hypercoagulability, and bone marrow failure. The characteristic defect in paroxysmal nocturnal haemoglobinuria is the somatic mutation of the PIG-A gene (essential to the synthesis of a moiety that interacts with a number of proteins on the cellular surface) in haematopoietic cells. These cells thus lack the proteins usually held in place by this "anchor". The current hypothesis explaining the disorder suggests that there are two components: (1) haematopoietic stem cells with the characteristic defect are present in the marrow of many if not all normal individuals in very small numbers; (2) some aplastogenic influence suppresses the normal stem cells but does not suppress the defective stem cells, thus allowing the proportion of these cells to increase. Current research attempts to substantiate this hypothesis, which is clearly based on a darwinian interpretation: what causes PNH is not only the mutation (necessary as it is), but also a selection force consisting in the destruction of normal bone marrow cells and in a selective survival of mutated cells. ${ }^{22}$

\section{EVIDENCE ON DNA ADDUCTS}

The examples of bacteria and mice exposed to toxic environments suggest that a mutator phenotype might originate by selection of cells with a selective advantage and impairment of DNA repair. Such a theory might explain a series of epidemiological observations that otherwise are not easily explainable.

\section{DNA adducts in cancer cases and controls}

Table 1 shows that several studies (with one exception) reported higher levels of DNA adducts among cancer cases than among controls. The relative risk of developing bladder or lung cancer in subjects with adducts higher than the median level (or the detection level) were in the order of 5 to $6 .^{23}$ What is particularly puzzling is that the association between adducts and cancer was unaffected by adjustment for exposures such as tobacco smoke. A potential explanation is inherited or acquired susceptibility, which would characterise subjects prone to develop cancer; such susceptibility would essentially be attributable either to (a) an inherited defect of DNA repair, or (b) an acquired defect of DNA repair attributable to selection of specific cell clones. 
Fruit and vegetable consumption and DNA adducts In a study of bladder cancer Peluso et $a l^{24}$ reported that the association between case/control status and the level of adducts (below or above the median value) was stronger in the subjects who consumed less than two portions of vegetables per day (odds ratio $7.80 ; 95 \%$ confidence intervals 3.0 to 20.3) than in heavy consumers $(\mathrm{OR}=4.98$ for consumers of two portions per day; $\mathrm{OR}=2.0$ for consumers of three or more portions). Lack of antioxidants contained in fruit and vegetables might create a metabolic environment that facilitates the selection of clones with DNA repair defects; this would explain the stronger association between DNA adducts and cancer in low than in heavy consumers of fruit and vegetables.

\section{Diet and DNA}

There are several clues that diet may exert its effect on cancer risk by interacting with DNA metabolism. For instance, dietary fish oil reduces the incidence of activating G-to-A point mutations in the K-ras gene in the colonic mucosa of rats. This effect is consistent with the induction of MGMT (the enzyme mentioned above), which rapidly removes promutagenic O6-methylguanine DNA adducts. ${ }^{25}$

A series of studies conducted on folate deficiency show that this nutritional impairment involves DNA metabolism and repair. It can cause an imbalance in DNA precursors, uracil misincorporation into DNA, and chromosome breakage. ${ }^{26}$ In a study in rats, a significantly retarded rate of DNA excision repair was observed in the folate deficient animals. ${ }^{27}$

Thus, the effects of diet (particularly of low consumption of fruit and vegetables, possibly through the deficiency of folate) on the DNA metabolism seem also to be complex.

\section{Age at first exposure and DNA adducts}

A study on DNA adducts measured by P32-postlabelling conducted by Wiencke et $a l^{28}$ suggests that the impact of smoking on DNA adduct levels may be different in current and former smokers. In current smokers, recent smoking intensity (cigarettes smoked per day) was the most important variable. In former smokers, age at smoking initiation was inversely associated with DNA adduct levels. According to the authors, "the results in former smokers suggest that smoking during adolescence might produce physiological changes that lead to increased DNA adduct persistence." Similar observations have been made by Vulimiri et al. ${ }^{29}$ They found that cases with lung cancer who started smoking at an earlier age had the highest levels of aromatic DNA adducts and 8-oxo-dG. Again, a potential explanation might be clonal selection of cells that are defective in DNA repair because of the environmental mutagenic agent (smoking), or because smoking acts as a positive selective agent on DNA repair deficient cells with an advantageous growth, or for both reasons.

\section{An experiment}

Bulky adduct forming (BAF) mutagens, comprising dietary components, pollutants, and intrinsic metabolites appear as primary candidates for a selection pressure that promotes CIN (chromosomal instability). A relation between the CIN phenotype and BAF mutagens is now demonstrated in a model system, tested in an experiment conducted by Bardelli and colleagues ${ }^{30}$ with the food borne carcinogen PhIP. As it has been proposed that the type of genetic instability in cancer cells reflects the selection pressures exerted by specific carcinogens, the researchers tested this hypothesis by treating immortal, genetically stable human cells with different carcinogens. They found that cells resistant to 2-amino-1methyl-6-phenylimidazo[4,5-b]pyridine (PhIP) exhibited CIN, whereas cells resistant to the methylating agent $\mathrm{N}$-methyl-N'-nitro-N-nitrosoguanidine (MNNG) exhibited microsatellite instability (MIN) associated with mismatch repair defects. Conversely, they found that cells purposely made into CIN cells were resistant to PhIP, whereas MIN cells were resistant to MNNG. PhIP is a diet borne carcinogen that causes the formation of "bulky" adducts, and induces colon and breast cancer in rats and mice.

\section{THE FINGERPRINT THEORY REVISITED}

We can propose a different explanation of the apparent "fingerprints" observed in cancer cells in conjunction with certain exposures. In fact, in some circumstances they can be the manifestation of selective survival of mutated clones. If this is true, the specificity of mutations (for example, the $\mathrm{T}$ to $\mathrm{G}$ transversions in codon 249 of $p 53$ ) might be related more to the metabolically altered environment - that exerts a selective pressure- than to the original mutagenic exposures. The following are a few tentative examples of how exposures of interest to epidemiologists might be responsible for the selection of clones carrying specific mutations.

1 Porta and colleagues ${ }^{31}$ have reported that patients with pancreatic cancer had mutations in the ras gene more frequently if they were regular coffee drinkers than nondrinkers. Coffee drinking was equally associated with the two main types of K-ras mutations-that is, there was no specific association with mutational spectrum. Coffee is known to impair DNA repair; thus, cells with ras mutations (induced by smoking or by other pancreatic carcinogens) might acquire a selective advantage in coffee drinkers through a variety of mechanisms.

2 Several types of evidence indicate that the Western diet is associated with chronic stimulation of pancreatic cells that produce insulin and with peripheral insulin resistance. Hyperinsulinaemia has been proposed as a risk factor for colon cancer $^{32}$ by exerting a proliferative stimulus on colon cells; in fact, it might act by selecting mutated clones.

3 We have observed that DNA adducts in bladder cells were higher in association with high tumour grades, ${ }^{33}$ suggesting that cancer development can lead to "tolerance" towards DNA damage, through clonal cell selection.

4 In the case of liver carcinoma, we have already mentioned that the selection of clones with $p 53$ mutations might be attributable to the action of the hepatitis B virus.

Concerning point 2 (hyperinsulinaemia), a rather articulate theory has been developed to explain the high frequency in Western populations of the so called "X syndrome", an association of obesity, hypertension, diabetes, and cardiovascular disease..$^{34}$ The theory says that our metabolic "make up" dates back to the Paleolithic human, who developed (like other animals) a "thrifty genotype": that is, in conditions of scarcity, his/her body tended to accumulate as much energy as possible. In addition, the Paleolithic human consumed much more antioxidants and other protective agents than today's Western humans (table 2). In the current circumstances of abundancy, the thrifty genotype has become a cause of chronic diseases, particularly if energy rich food is consumed with comparatively poor intake of protective agents (table 2 ). ${ }^{35}$

The most evident example of the theory is represented by migrants or people who have quickly changed their dietary habits from a quasi-Paleolithic type to a Western type, in particular Pima Indians in Southern Arizona. They had to adapt to a high fat, highly refined, government dispensed diet after abandoning their agricultural work. Currently Pima adults in Southern Arizona have a mean BMI of 33.4, while 37\% of men and $54 \%$ of women meet the criteria for NIDDM (diabetes); this strongly contrasts with the traditional Pima who live in Northern Mexico. The causal complex that has led to such a situation is a gene-environment interaction that entails a rapid change of dietary habits in an "unprepared" person. Displacement of people, dietary habits that change quickly as a consequence of market trends, loss of jobs, all are characteristic features in the life of the Pima indians and of millions of other people on the earth. 
Table 2 Typical intake of different nutrients by the Paleolithic humans and contemporary Western humans (from McCully ${ }^{35}$ )

\begin{tabular}{lll}
\hline & Paleolithic & $\begin{array}{l}\text { Contemporary } \\
\text { (Western) }\end{array}$ \\
\hline Animal proteins (\% of calories) & 33 & $12-14$ \\
Fats (\% of calories) & $20-25$ & $>30$ \\
Saturated fats (\% of calories) & 6 & 14 \\
Folate & $357 \mu \mathrm{g} / \mathrm{g}$ & $150-200 \mu \mathrm{g} / \mathrm{g}$ \\
Vitamin B6 & $3 \mathrm{mg} / \mathrm{g}$ & $1.5 \mathrm{mg} / \mathrm{g}$ \\
Vitamin B12 & $15 \mu \mathrm{g} / \mathrm{g}$ & $9 \mu \mathrm{g} / \mathrm{g}$ \\
Ascorbic acid & $600 \mathrm{mg} / \mathrm{g}$ & $77-109 \mathrm{mg} / \mathrm{g}$ \\
Fibre & $104 \mathrm{~g} / \mathrm{g}$ & $10-20 \mathrm{~g} / \mathrm{g}$ \\
Sodium & $0.7 \mathrm{~g} / \mathrm{g}$ & $>6 \mathrm{~g} / \mathrm{g}$ \\
\hline
\end{tabular}

These are only a few examples that suggest that the hypothesis of clone selection can be a way to integrate seemingly disparate epidemiological observations under a common theoretical framework. At least three categories of epidemiological risk factors can be included in such a model: dietary exposures, hormones, and infections. "Growth factors", such as IGF1 or TGF $\alpha$ or $\beta$, could be involved in such mechanisms.

In agreement with the present interpretation, Steen has made mathematical calculations that lead to exclude that cancer arises from a single cell. Rather, "we are led to conclude that the primary events may take place in other cells than the one which eventually becomes initiated, which implies that intercellular interactions may have a crucial role in the etiology of common cancer". ${ }^{36}$ According to Steen, collective, intercellular processes, or rather the interruption of such processes, may be essential in carcinogenesis. The processes alluded to can be modified by signals like growth factors, hormones, or electrochemical interactions. An interesting concept introduced by Steen is that of "local collapse": "a mutation in any gene of any cell may, however little, contribute to a degradation of the integrity of the system . . . The result of such degradation could be a local collapse of the system, leading to a drastic increase of the probability of an initiating oncogenic event in that locality". ${ }^{36}$ The similarities between this description and the example of paroxysmal nocturnal haemoglobinuria is striking.

\section{THE EXAMPLE OF ARSENIC}

What can be the advantage of this "darwinian" interpretation? In addition to providing a theoretical framework for epidemiology, it may help to find a potential explanation for conflictual observations. We give one example here.

Arsenic is a rather unusual toxicant, as it causes different diseases in different populations: lung cancer in foundry workers $^{37}$; typical proliferative lesions of the skin of the hands, cancer of the skin and of the bladder in the inhabitants of Antofagasta, an area in Chile where contamination of water by arsenic is endemic ${ }^{38}$; and the so called "black foot disease" (a peripheral arteriopathy) in Taiwan, again as a consequence of water contamination. ${ }^{39} \mathrm{~A}$ way to interpret these contradictory findings is to assume that different populations have been exposed to different mutagens before undergoing exposure to arsenic, and that arsenic selects mutated cell clones. In studies of foundry workers, arsenic seemed to act as a late stage carcinogen, thus supporting the hypothesis we suggest. ${ }^{40}$ As arsenic changes the cell metabolism, chronic exposure can create a metabolic environment that allows mutated cells to acquire a selective advantage. Recent evidence suggests that arsenic stimulates the production of growth factors such as TGF $\alpha$ and the epidermal growth factor. ${ }^{41}{ }^{42}$ In addition, in a rather complex way arsenic is involved also in the metabolism of folate: arsenic is detoxified through methylation by a methyltransferase; arsenic methylation subtracts methyl groups to

\section{Key points}

- Mutations seem to be only one of the mechanisms involved in carcinogenesis; selection of mutated clones is a second crucial mechanism.

- In both the selection of species and in carcinogenesis (selection of mutated cells), mutation and selection can be interpreted as necessary and insufficient causes. Selection presupposes competition among clones-that is, survival advantage of the mutated species; without selective forces a mutation is mute, while the lack of mutations makes selective advantage impossible.

- The identification of carcinogen related fingerprints is ambiguous: it can suggest both a genuine mutational hotspot left by the carcinogenic stimulus (like in tobacco related p53 mutations), and selective advantage of clones whose mutations seem to be not exposure specific (like in the case of aflatoxin).

- A disease model is paroxysmal nocturnal haemoglobinuria. Current research attempts to substantiate the hypothesis, clearly based on a darwinian interpretation, that what causes PNH is not only a mutation of the PIG-A gene (which is necessary), but also a selection force consisting in the destructioin of normal bone marrow cells and in a selective survival of mutated cells.

- An evolutionary interpretation of carcinogenesis can shed light on contradictory findings of epidemiology.

MTHFR, the polymorphic enzyme that metabolises folate. Therefore, arsenic might indirectly cause folate deficiency. ${ }^{43}$ Finally, and most important, there is good evidence that arsenic impairs DNA repair. In particular, in ovary cells irradiated with ultraviolet light or incubated with MMS (that induce strand breaks), treatment with arsenic inhibited postreplication repair. ${ }^{44}$ In other experiments, arsenic inibited the excision of typical ultraviolet induced pyrimidine dimers. ${ }^{44}$ The inhibition of repair seems to be related to an effect on Ligase II. ${ }^{44}$

\section{MATHEMATICAL MODELS OF CLONE SELECTION}

The mathematical relations between time dependent exposures, such as tobacco smoke, and cancer have been proposed according to two main models, the Armitage-Doll "multistage" model and Moolgavkar's two stage model. In both cases, cancer is basically interpreted as the consequence of a sequence of mutations, although Moolgavkar provides a term for cell replication/clonal selection. However, conceiving carcinogenesis as a darwinian process might imply the use of a completely different set of equations, for example Lotka and Volterra's models used to describe the competition among species for the same habitat. Some work has already been done by using Lotka-Volterra mathematical models, but only on theoretical gounds. ${ }^{45}$ 


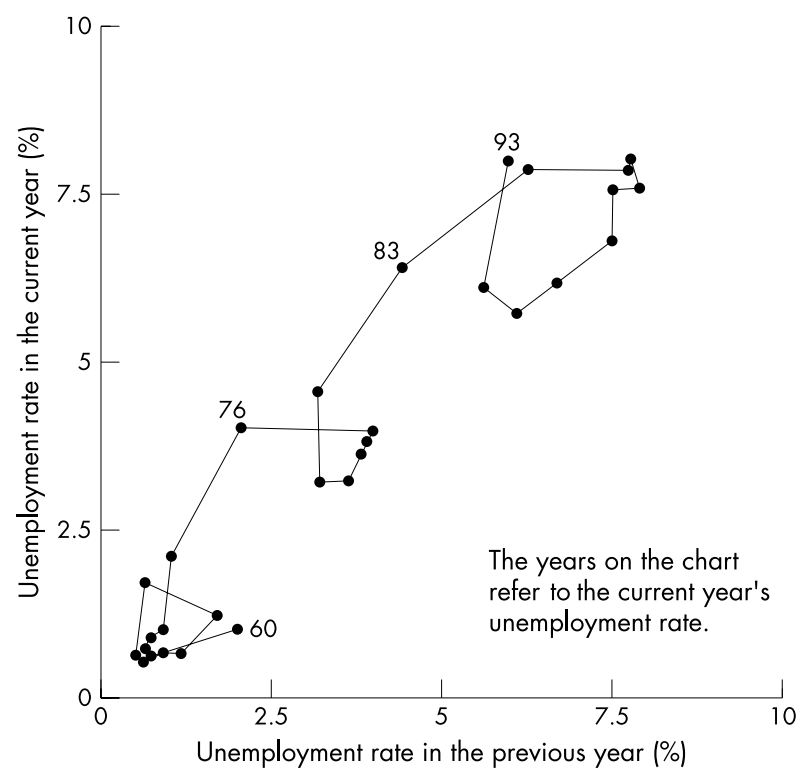

Figure 1 Concentrated scatter plot of West German unemployment rate, 1960-93.

A similarity can help understanding why new mathematical models could prove useful in carcinogenesis. Figures 1 and 2 show two examples of the relation between unemployment in a certain year and unemployment in the previous year $\left(\right.$ from Ormerod $\left.^{46}\right)$. In West Germany unemployment was characterised by three cycles, each of which represents a homeostatic mechanism, while in Sweden only one cycle was followed by a dramatic linear increase of unemployment. The "force" that explains the homeostatic mechanism is called "attractor": in Sweden a very strong attractor existed, at low levels of unemployment, until 1991, and then disappeared. In West Germany, the attractor was weak and acted at different stages of the process. The metaphor of the attractor can be used in carcinogenesis to refer, for instance, to DNA repair and other similar homeostatic mechanisms, which, we hypothesise, are particularly important at low levels of exposure to damaging agents.

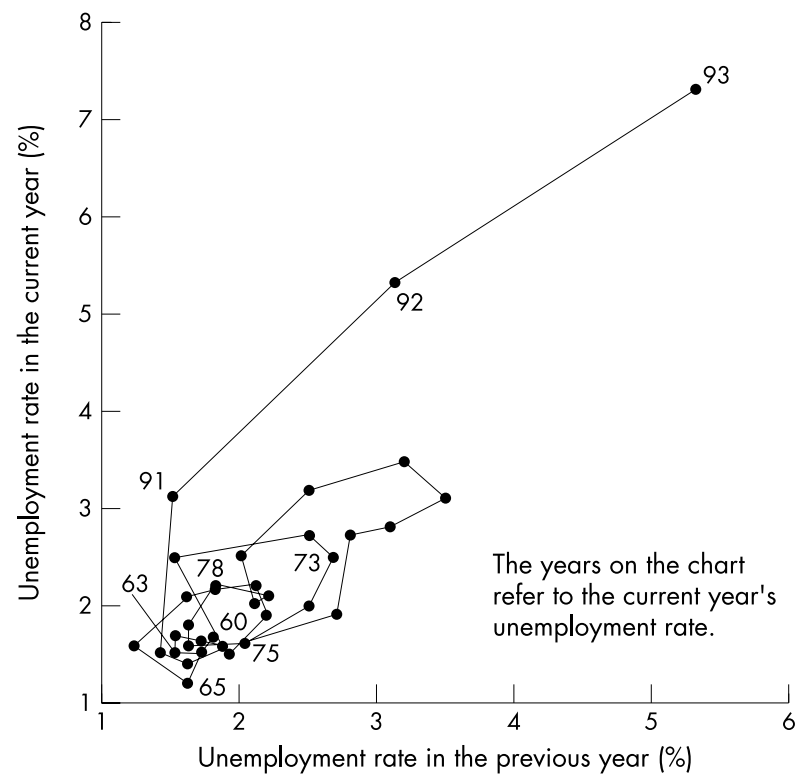

Figure 2 Concentrated scatter plot of Swedish unemployment rate, 1960-93.
Lotka-Volterra's equations describe similar phenomena when dealing with the relations between two different populations (including cells) competing for the same territory. Again, a steady state can be altered by small changes that overcome the homeostatic mechanisms.

\section{CONCLUSIONS}

A darwinian theory of carcinogenesis can be useful to understand some contradictory observations of epidemiology and to provide a common theoretical framework for carcinogenesis. In both speciation and carcinogenesis, mutation and selection can be interpreted as necessary and insufficient causes. Selection presupposes competition among clones, and a survival advantage of the mutated species; therefore, without selective forces a mutation is mute, while the lack of mutations makes selective forces inconsequential. The identification of carcinogen related fingerprints may sometimes be misleading, as it can reflect a genuine mutational hotspot left by the carcinogenic stimulus (like in tobacco related $p 53$ mutations) or the selective advantage of clones whose mutations seem to be not exposure specific (like in the case of aflatoxin).

Unfortunately, epidemiology has some important limitations. In particular, we tend to study the prevalence of events such as mutations at a given time. The epidemiological design is still far from allowing researchers to study the time sequence and the interplay of complex events, because only few measurements are currently feasible in large human populations.

\section{ACKNOWLEDGEMENT}

We are grateful to Douglas Weed, David Berrigan, Stefano Bonassi, Tony McMichael, Paola Pisani and David De Marini for thoughtful comments. A special thanks goes to Miquel Porta for his brilliant contribution.

\section{Authors' affiliations}

P Vineis, CPO-Piemonte and Università di Torino, Italy

P Vineis, G Matullo, M Manuguerra, Institute for Scientific Interchange

Foundation, Torino, Italy

G Matullo, Dipartimento di Genetica, Università di Torino

Funding: this paper has been made possible by a grant from the European Commission (Vth Network Programme) for the "Gen-Air" project (contract no QLK4-1999-00927) and a grant from the World Cancer Research Fund.

\section{REFERENCES}

1 Vineis $\mathbf{P}$, Schulte $\mathrm{P}, \mathrm{McMichael}$ AJ. Misconceptions about the use of genetic tests in populations. Lancet 2001;357:709-12.

2 Strohman RC. The coming Kuhnian revolution in biology. Nat Biotechnol 1997; 15:194-200.

3 Vineis $\mathbf{P}$, Malats N, Porta $M$, et al. Human cancer, carcinogenic exposures and mutational spectra. Mutation Res 1999;436:185-94

4 Haddow A. Sir Ernest Laurence Kennaway FRS, 1881-1958: chemical causation of cancer then and today. Perspect Biol Med 1974;17:543-88.

5 Lawley PD, Brookes P. Molecular mechanism of the cytotoxic action of difunctional alkylating agents and of resistance to this action. Nature 1965;206:480-3

6 Hussain SO, Hollstein M, Harris CC. p53 tumor suppressor gene: at the crossroads of molecular carcinogenesis, molecular epidemiology, and human risk assessment. Ann N Y Acad Sci 2000;919:79-85.

7 Denissenko MF, Koudriakova TB, Smith L, et al. The p53 codon 249 mutational hotspot in hepatocellular carcinoma is not related to selective formation or persistence of aflatoxin B1 adducts. Oncogene 1998;17:3007-14.

8 Denissenko MF, Pao A, Tang M, et al. Preferential formation of benzo[a]pyrene adducts at lung cancer mutational hotspots in P53. Science 1996;274:430-2.

9 Rodin SN, Rodin AS. Human lung cancer and p53: the interplay between mutagenesis and selection. Proc Natl Acad Sci USA 2000:97:12244-9.

10 Hainaut $\mathbf{P}$, Pfeifer GP. Patterns of $\mathrm{p} 53 \mathrm{G}$ to $T$ transversions in lung cancers reflect th eprimary mutageni signature of DNA-damage by tobacco smoke. Carcinogenesis 2001;22:367-74.

11 Jonason AS, Kunala S, Price GJ, et al. Frequent clones of p53-mutated keratinocytes in normal human skin. Proc Natl Acad Sci USA 1996;93:14025-9. 
12 Iversen $\mathrm{OH}$. The skin tumorigenic and carcinogenic effects of different doses, number of dose fractions and concentrations of 7,12-dimethylbenz(a)anthracene in acetone applied in hairless mouse epidermis. Possible implications for human carcinogenesis. Carcinogenesis 1991;12:493-502.

13 International Agency for Research on Cancer. IARC Monographs on the evaluation of the carcinogenic risk of chemicals to humans. Vol 38. Tobacco smoking. Lyon: IARC, 1986.

14 Eldredge N. Reinventing Darwin. The great debate at the high table of evolutionary theory. New York: Wiley, 1995.

15 Leigh EG. The evolution of mutation rates. Genetics 1973;73:1-18.

16 Karran P, Bignami M. DNA damage tolerance, mismatch repair and genomic instability. Bioessays 1994; 16:833-9.

17 Taddei F, Radman M, Maynard-Smith J, et al. Role of mutator alleles in adaptive evolution. Nature 1997:387:700-2.

18 LeClerc JE, Li B, Payne WL, et al. High mutation frequencies among Escherichia coli and Salmnonella pathogens. Science 1996;274:120811.

19 Haraguchi Y, SasakiA. Host-parasite arms race in mutation modifications: indefinite escalation despite a heavy load? J Theor Biol 1996; 183:121-37.

20 Tomlinson I, Bodmer W. Selection, the mutation rate and cancer: ensuring that the tail does not wag the dog. Nat Med 1999;5:11-12.

21 Rowan AJ, Lamlum $H$, llyas $M$, et al. APC mutations in sporadic colorectal tumours: a mutational "hotspot" and interdependence of the "two hits". Proc Natl Acad Sci USA 2000;97:3352-7.

22 Rosse WF. New insights into paroxysmal nocturnal hemoglobinuria Current Opinion in Hematology 2001;8:61-7.

23 Vineis $\mathbf{P}$, Perera F. DNA adducts as markers of exposure to carcinogens and risk of cancer. Int J Cancer 2000:88:325-8.

24 Peluso M, Airoldi L, Magagnotti C, et al. White blood cell DNA adducts and fruit and vegetable consumption in bladder cancer. Carcinogenesis 2000;21:183-7.

25 Davidson LA, Lupton JR, Jiang YK, et al. Carcinogen and dietary lipid regulate ras expression and localization in rat colon without affecting farnesylation kinetics. Carcinogenesis 1999;20:785-91.

26 Duthie SJ, Narayanan S, Blum S, et al. Folate deficiency in vitro induces uracil misincorporation and DNA hypomethylation and inhibits DNA excision repair in immortalized normal human colon epithelial cells. Nutr Cancer 2000;37:245-51.

27 Choi SW, Kim Yl, Weitzel JN, et al. Folate depletion impairs DNA excision repair in the colon of the rat. Gut 1998;43:93-9.

28 Wiencke JK, Thurston SW, Kelsey KT, et al. Early age at smoking initiation and tobacco carcinogen DNA damage in the lung. J Natl Cancer Inst 1999;91:614-19.

29 Vulimiri SV, Wu X, Baer-Dubowska W, et al. Analysis of aromatic DNA adducts and 7,8-dihydro-8-oxo-2' deoxyguanosine in lymphocyte DNA from a case-control study of lung cancer involving minority populations. Mol Carcinog 2000;27:330.

30 Bardelli A, Cahill DP, Lederer G, et al. Carcinogen-specific induction of genetic instability. Proc Natl Acad Sci USA 2001;98:5770-5.

31 Porta M, Malats N, Alguacil J, et al. Coffee, pancreatic cancer, and K-ras mutations: updating the research agenda. J Epidemiol Community Health 2000;54:656-9.

32 Nilsen TI, Vatten L. Prospective study of colorectal cancer risk and physical activity, diabetes, blood glucose and BMI: exploring the hyperinsulinaemia hypothesis. Br J Cancer 2001;84:417-22.

33 Airoldi I, Orsi F, Magagnotti C, et al. Determinants of 4-aminobiphenyl-DNA adducts in bladder cancer biopsies. Carcinogenesis 2002;23:861-6.

34 Neel JV, Weder AB, Julius S. Type II diabetes, essential hypertension and obesity as "Syndromes of impaired genetic homeostasis": the "thrifty genotype" hypothesis enters the $21^{\text {st }}$ century. Persp Biol Med 1998:42:44-75

35 McCully KS. The significance of wheat in the Dakota territory, human evolution, civilization, and degenerative diseases. Persp Biol Med 2001;44:52-61.

36 Steen HB. The origin of oncogenic mutations: where is the primary damage? Carcinogenesis 2000;21:1773-6.

37 International Agency for Research on Cancer. IARC Monographs on the evaluation of the carcinogenic risk of chemicals to humans. Vol. 23. Some metals and metallic compounds. Lyon: IARC, 1986.

38 Smith AH, Goycolea M, Haque R, et al. Marked increase in bladder and lung cancer mortality in a region of Northern Chile due to arsenic in drinking water. Am J Epidemiol 1998;147:660-9.

39 Ko YC. A critical review of epidemiologic studies on black-foot disease. Journal of the University of Occupational and Environmental Health 1986;8:339-53.

40 Brown CC, Chu KC. Implications of the multistage theory of carcinogenesis applied to occupational arsenic exposure. J Natl Ccancer Inst 1983;70:455-63.

41 Germolec DR, Spalding J, Yu HS, et al. Arsenic enhancement of skin neoplasia by chronic stimulation of growth factors. Am J Pathol 1998;153:1775-85.

42 Wu W, Graves LM, Jaspers I, et al. Activation of the EGF receptor signaling pathway in human airway epithelial cells exposed to metals. Am J Physiol 1999;277:L924-31.

43 Van Tongeren JH, Kunst A, Majoor CL, et al. Folic-acid deficiency in chronic arsenic poisoning. Lancet 1965; i:784-6

44 Basu A, Mahata J, Gupta S, et al. Genetic toxicology of a paradoxical human carcinogen, arsenic: a review. Mut Res 2001;488:171-94.

45 Gatenby RA. Application of competition theory to tumour growth: implications for tumour biology and treatment. Eur J Cancer 1996;32A:722-6.

46 Ormerod P. The death of economics. New York: Wiley, 1997. 